\title{
Cryptogenic haemoptysis in smokers: angiography and results of embolisation in 35 patients
}

\author{
L. Menchini*\#, M. Remy-Jardin*, J-B. Faivre*, M-C. Copin`, P. Ramon+, R. Matran, \\ V. Deken ${ }^{f}$, A. Duhamel ${ }^{f}$ and J. Remy*
}

ABSTRACT: The aim of the present study was to describe angiographic findings and embolisation results in smokers with haemoptysis.

We retrospectively reviewed the clinical data and angiographic findings from 35 patients with smoking-related bronchopulmonary disease and no associated comorbidity, who were referred for embolisation for mild $(n=6)$, moderate $(n=14)$ and severe $(n=15)$ haemoptysis. Spirometric classification subdivided our population into: 16 patients with chronic bronchitis but no airflow limitation; and 19 patients with chronic obstructive pulmonary disease (COPD) (stage I: $n=12$; stage II: $n=5$; stage III: $n=2$ ).

Bronchoscopy depicted focal submucosal vascular abnormalities in three patients and only endobronchial inflammation in $32(91 \%)$ patients. Bronchial artery angiography revealed moderate $(n=18)$ or severe $(n=10)$ hypervascularisation in $28(80 \%)$ patients, and normal vascularisation in seven $(20 \%)$. No statistically significant difference was observed between the angiographic findings and the severity of COPD, tobacco consumption or the amount of bleeding. Cessation of bleeding was obtained by embolisation in 29 out of the $\mathbf{3 4}$ technically successful procedures $(85 \%)$, requiring surgery in three out of five patients with recurrence. Follow-up (mean duration $7 \mathrm{yrs}$ ) demonstrated no recurrence of bleeding in 32 (94\%) out of 34 patients and excluded late endobronchial malignancy.

Smokers with various stages of COPD severity may suffer from haemoptysis that is efficiently treatable by endovascular treatment.

KEYWORDS: Bronchial arteries, chronic obstructive pulmonary disease, smoker's lung

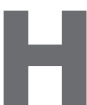

aemoptysis is a frequent symptom in pneumology and requires rapid and careful investigation. The changing spectrum of causes of haemoptysis was first acknowledged in the late 1980s by the study of JOHNSTON and REISz [1]. Comparing their data with older studies, they underlined that haemoptysis was less likely to be caused by tuberculosis and bronchiectasis, while haemoptysis caused by bronchitis had increased proportionately, subsequently confirmed in the literature [1-6]. The second striking feature regarding the revised prevalence of the aetiologies of haemoptysis is that, despite modern diagnostic means, nearly a third of haemoptysis remains classified as cryptogenic [1, 3, 7-10]. However, the populations reported in this category are quite heterogeneous, gathering patients with no identifiable causes of haemoptysis, but also patients in whom an underlying disease was later recognised, such as "inactive tuberculosis" or "occult bronchiectasis". This apparent contradiction can be explained by the various criteria retained by the authors to assess this diagnosis. If fibreoptic bronchoscopy was systematically included [1, 3, 7-10], patients' follow-up and imaging studies, in particular chest computed tomography (CT), have been only integrated in the most recent reports on cryptogenic haemoptysis $[3,6,9,10]$.

When no associated comorbidity can be confidently excluded, the subgroup of cryptogenic haemoptysis shares a common risk factor with patients in whom haemoptysis is explained by chronic bronchitis, namely the patient's tobacco consumption. Careful reading of studies on cryptogenic haemoptysis in which detailed patient histories are available reveals a high proportion of smokers, varying between $42 \%$ and $79 \%$ of the studied populations [2, 8-10]. However, these authors did not discuss the causal relationship between cryptogenic haemoptysis and smoking, as did Hiyama et al. [11] in 2002. In our experience
AFFILIATIONS

Depts of *Thoracic Imaging (EA 2694),

+Pulmonology, and

${ }^{\S}$ Pulmonary Function, Hospital Calmette, University Centre of Lille, Depts of "Pathology, and,

${ }^{f}$ Medical Statistics (EA 2694),

University Center of Lille, Lille Cedex, France.

\#Dept of Radiology Paediatric Hospital "Bambino Gesù", Rome, Italy.

CORRESPONDENCE

M. Remy-Jardin

Dept of Thoracic Imaging

Hospital Calmette

Boulevard Jules Leclercq

59037 Lille Cedex

France

E-mail: mremy-jardin@chru-lille.fr

Received:

Feb 042009

Accepted after revision:

March 162009

First published online

April 082009 
of a referral centre for embolisation of haemoptysis, we encountered similar situations in which the patient's smoking history was the only potential cause for bronchial bleeding after a thorough search for an underlying disease. The purpose of this study was to report the angiographic aspects of the bronchial circulation and the long-term results of bronchial artery embolisation in this subset of patients with various stages of smoking-related disease and no other comorbidity.

\section{MATERIALS AND METHODS}

\section{Study population}

The study population was selected from the database of 318 consecutive patients who had been referred to the Department of Radiology for endovascular treatment of haemoptysis over a period of 22 yrs (November 1983 to February 2006). The criteria for selecting this population included: 1) the absence of any comorbidity when assessed by fibreoptic endoscopic examinations and imaging studies at the time of admission; 2) clinical symptoms of chronic bronchitis in a context of smoking-history; 3) no late diagnosis of bronchial carcinoma confirmed by patients' follow-up. Out of 318 patients, 35 (11\%) fulfilled these criteria. None of these patients showed clinical and/or biological features of concomitant infection at the time of their referral for haemoptysis. Except for two patients, for whom the information was not retrospectively accessible, our population did not receive aspirin or coumadin treatment when evaluated for bronchial bleeding. All patients were first treated medically to try to stop bronchial bleeding; if it did not, the embolisation procedure was undertaken.

\section{Fibreoptic bronchoscopy}

Fibreoptic bronchoscopy was performed within $48 \mathrm{~h}$ of admission in cases of mild to moderate haemoptysis or as an emergency in cases of massive haemoptysis. The bronchoscopic report stated: 1) the presence of active bleeding; 2) the site of bleeding and, whenever possible, the lobar origin of bleeding; and 3) the presence of endobronchial abnormality. Endobronchial and transbronchial biopsies were performed when indicated, and all specimens were routinely examined for cytology and microbiology.

\section{Imaging studies}

At the time of admission, each patient underwent a radiographic examination of the chest. To exclude an underlying lesion potentially responsible for bronchial bleeding, patients underwent additional imaging studies, consisting of conventional bronchography $(n=6)$ and/or tomography $(n=4)$ in the nine $(26 \%)$ patients referred prior to the advent of CT, and chest CT in the remaining $26(74 \%)$ patients. Depending on the CT technology available, high-resolution chest CT was obtained using a single- $(n=12)$, four- $(n=5), 16-(n=4)$ and 64- $(n=5)$ slice CT scanner. Initial and follow-up CT scans were reviewed to exclude any specific anatomic lesion as the source of bleeding and to describe the presence of alveolar infiltration and/or endobronchial nodules, reflecting the filling of the alveolar spaces and airways with blood, respectively. No attempt was made to describe the $\mathrm{CT}$ appearance of bronchial arteries in our study group because CT was not always available (nine out of 35 studied patients) or consisted of a noncontrast CT scan (13 out of 26 patients with CT).

\section{Angiography}

In all patients, conventional angiography, with a Seldinger technique, was performed with a conventional, then digital subtraction technique within one week of admission (range 17 days). Management of haemoptysis followed the general recommendation according to which bronchial arteries ipsilateral to the side of bronchial bleeding should be embolised first [12]. Before the embolisation procedure, a bronchial angiogram was systematically performed with manual injection of contrast medium in the catheterised vessel, enabling analysis of the diameter of bronchial arteries and depiction of angiographic features of hypervascularisation on the side of bleeding. The diameter of bronchial arteries was coded as normal when $<2 \mathrm{~mm}$, moderately enlarged when the diameter was $2-4 \mathrm{~mm}$ and severely dilated when the diameter was $>4 \mathrm{~mm}$. The additional angiographic features of hypervascularisation included the depiction of: 1) tortuous enlargement of bronchial arteries that supplied an area of parenchymal staining; and 2) a shunt into pulmonary vessels. Bronchial vascularisation was coded as normal, moderately or markedly increased. Endobronchial bleeding occurring during the embolisation procedure was systematically recorded. The angiographic appearance of controlateral bronchial arteries was available in two conditions: 1) when the side of bleeding was supplied by a common trunk for the right and left bronchial arteries; and 2) when controlateral bronchial arteries were incidentally catheterised during the angiographic procedure. When controlateral bronchial angiograms were available, the diameter of the bronchial arteries and the presence of angiographic features of hypervascularisation were coded using the same criteria as those used for the analysis on the side of bleeding.

Persistent haemoptysis after a technically successful procedure required a verification of the status of previously embolised vessels: 1) in the case of partial recanalisation, the arteries were again embolised; and 2) in the case of persistent adequate obstruction, then recurrence of bleeding constituted an indication for evaluation of nonbronchial systemic arteries on the side of bleeding. This procedure was always considered at second intention because of the potential neurological iatrogenic risks when embolising these vessels and the lack of constant relationship between arterial hypervascularity and bronchial bleeding. The immediate and long-term results of embolisation were systematically recorded.

\section{Imaging study interpretation}

The imaging studies undertaken at the time of the initial management were reviewed by two radiologists (L. Menchini and M. Remy-Jardin), with 5 and 20 yrs of experience in thoracic imaging, respectively, who were both unaware of the patient's clinical condition at the time of their reading. When initial and follow-up CT scans were available, they were analysed consecutively by the two readers to assess the outcome of CT findings.

\section{Statistical analysis}

Statistical analysis was performed with commercially available software (SAS Institute, Cary, NC, USA). Results were expressed as mean $\pm \mathrm{SD}$ and ranges for continuous variables, and as frequencies and percentages for categorical variables. Comparative 
TABLE 1 Amount of bronchial bleeding according to the spirometric classification of the 35 patients

\begin{tabular}{|c|c|c|c|c|}
\hline & Mild haemoptysis & Moderate haemoptysis & Massive haemoptysis & Total \\
\hline Smokers with no airflow limitation & 2 & 8 & 6 & 16 \\
\hline Smokers with moderate or severe COPD & 1 & 3 & 3 & 7 \\
\hline Total & 6 & 14 & 15 & 35 \\
\hline
\end{tabular}

analyses were obtained using the Chi-squared or the Fisher exact test for categorical data. For numerical variables, group comparisons (if the sample was >30) were performed using either the Mann-Whitney U-test (when there were two groups) or the Kruskal-Wallis test (when there were more than two groups). A p-value $<0.05$ was considered statistically significant.

\section{RESULTS}

\section{Characteristics of the population studied}

Clinical data

The investigated cohort included 33 males and two females (mean age $57.08 \pm 10.6 \mathrm{yrs}$; range $34-76 \mathrm{yrs}$ ) and a mean cigarette consumption of $33.4 \pm 14.54$ pack-yrs (range 12 80 pack-yrs). The absence of an underlying anatomic lesion as the source of bleeding was assessed in all patients by the review of imaging findings (chest films and CT scans in 26 (74\%) patients; chest films, conventional bronchography and/ or tomography in nine (26\%) patients). The mean follow-up was $7.01 \pm 6.8$ yrs (range 1.6-26.8 yrs). The absence of underlying lung cancer was assessed on the basis of: 1) an uneventful mean follow-up period of $9.72 \mathrm{yrs}$ in the nine patients who did not undergo chest CT at the time of initial management; and 2) an uneventful mean follow-up period of $6.07 \mathrm{yrs}$ in 26 patients who underwent chest CT at the time of initial management; among them, 24 patients were followed-up by fibreoptic bronchoscopy and chest CT which did not reveal any tumoural lesion.

Applying the revised criteria of the Global Initiative for Chronic Obstructive Lung Disease (GOLD) strategy [13], our population included: 1) 16 patients with symptoms of chronic bronchitis but no airflow limitation (i.e. healthy smokers); and 2) 19 patients with chronic obstructive pulmonary disease (COPD) (stage I: $\mathrm{n}=12$; stage II: $\mathrm{n}=5$; stage III: $\mathrm{n}=2$ ). The amount of haemoptysis was mild $\left(<100 \mathrm{~mL} \cdot\right.$ day $\left.^{-1} ; \mathrm{n}=6\right)$, moderate $\left(100-200 \mathrm{~mL} \cdot\right.$ day $^{-1}$; $\mathrm{n}=14)$ or massive $\left(>200 \mathrm{~mL} \cdot \mathrm{day}^{-1} ; \mathrm{n}=15\right)$. In all patients, bronchial embolisation was indicated because of the amount and/or recurrence of bleeding despite medical treatment. Table 1 summarises the amount of bleeding in the four categories of patients classified according to the spirometric data. For the purpose of statistical analysis, we gathered the five patients with stage II COPD and the two patients with stage III COPD into the same subgroup; no statistically significant difference was observed in the distribution of the amount of bleeding between the three groups of smokers $(\mathrm{p}=0.74)$. For 21 patients, it was the first episode of haemoptysis, while 14 patients had already been admitted to our hospital for the treatment of mild $(n=9)$, moderate $(n=4)$ and severe $(n=1)$ bleeding, all stopped successfully with medical treatment.

\section{Fibreroptic bronchoscopic findings}

Active bleeding at the time of the endoscopic procedure or the focal location of blood clots led to the identification of the site of bronchial bleeding during fibreoptic bronchoscopy in all patients. The sites of bronchial bleeding were the upper lobes (25 out of 35; 71\%), with a right-sided predominance of upper lobe bleeding (18 out of $25 ; 72 \%$ ), the right middle lobe and lingula (two out of $35 ; 6 \%$ ) and the lower lobes (eight out of 35 ; $23 \%$ ). Fibreoptic bronchoscopy depicted the presence of mucosal inflammation in $32(91 \%)$ patients, localised to the area of bleeding in $20(62.5 \%)$ out of 32 patients or diffusely observed in the bronchial tree in $12(37.5 \%)$. In three $(9 \%)$ patients, fibreoptic bronchoscopy revealed the presence of submucosal vascular abnormalities in the area of bronchial

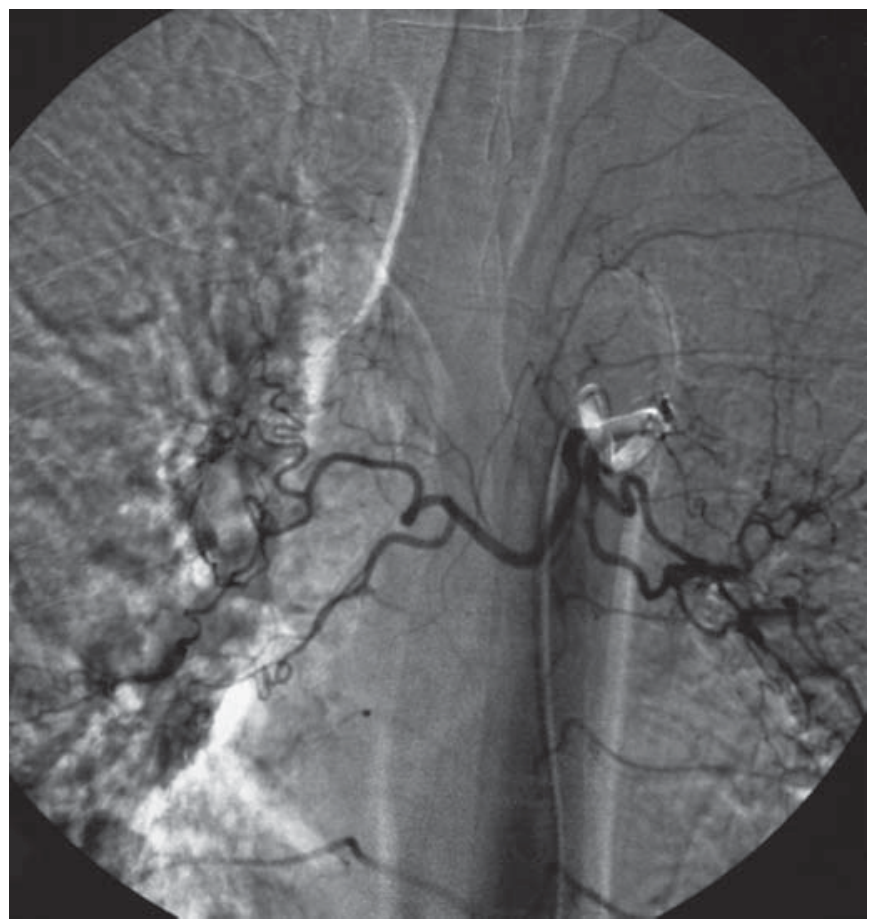

FIGURE 1. A 58-yr-old male with a cigarette consumption of 12 pack-yrs and no airflow limitation on pulmonary function tests. This patient was referred for management of moderate haemoptysis originating from the mediobasal segment of the right lower lobe. Selective catheterisation of a common trunk for the right and left bronchial arteries showing moderately dilated bronchial arteries on both sides. Embolisation of this artery led to an immediate cessation of haemoptysis that recurred 1 month later; a second embolisation procedure enabled definitive cessation of bronchial bleeding (9-yr follow-up) 


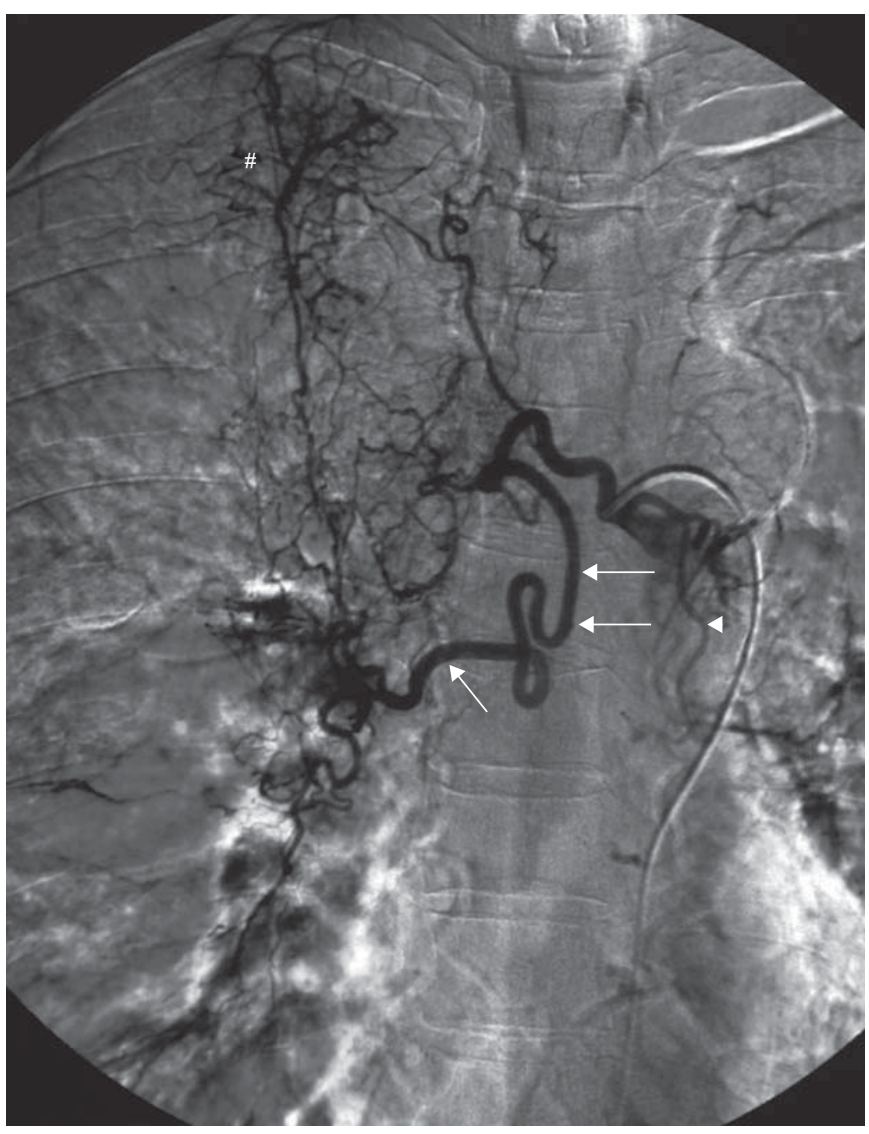

FIGURE 2. A 70-yr-old patient with a cigarette consumption of 69 pack-yrs and no airflow limitation on pulmonary function tests. This patient was referred for management of moderate haemoptysis originating from the right upper lobe. Selective catheterisation of the right intercostobronchial trunk showing features of marked hypervascularisation on the side of bleeding including a tortuous and dilated right bronchial artery (arrows) and bronchial-to-pulmonary retrograde shunts in the right apex (\#). Note the presence of tortuous but minimally dilated left bronchial branches (arrowhead), opacified because of the vicinity between the ostium of the left bronchial artery and that of the right intercostobronchial trunk. Immediate cessation of haemoptysis was obtained after embolisation of the right intercostobronchial trunk with no recurrence of bronchial bleeding over the 5-yr follow-up period.

bleeding, described as nonpulsating submucosal vascular nodules (two patients) or dilated submucosal vessels (one patient). In the overall population, no additional endobronchial abnormality or, in particular, no endobronchial malignancy was found during the endoscopic examination.

\section{Chest radiography and CT findings}

Chest radiography was normal in $17(54 \%)$ patients and abnormal in $16(46 \%)$ patients, showing alveolar infiltrates in 12 patients and emphysematous changes in four patients. Among the 26 patients who underwent chest $\mathrm{CT}$ at the time of referral for haemoptysis, CT was normal in two and depicted features of alveolar and/or endobronchial bleeding in 24 patients, which resolved on follow-up CT scans in all patients. An endobronchial nodule was found on the initial CT scan in 12 patients which resolved on the follow-up examination in all patients.

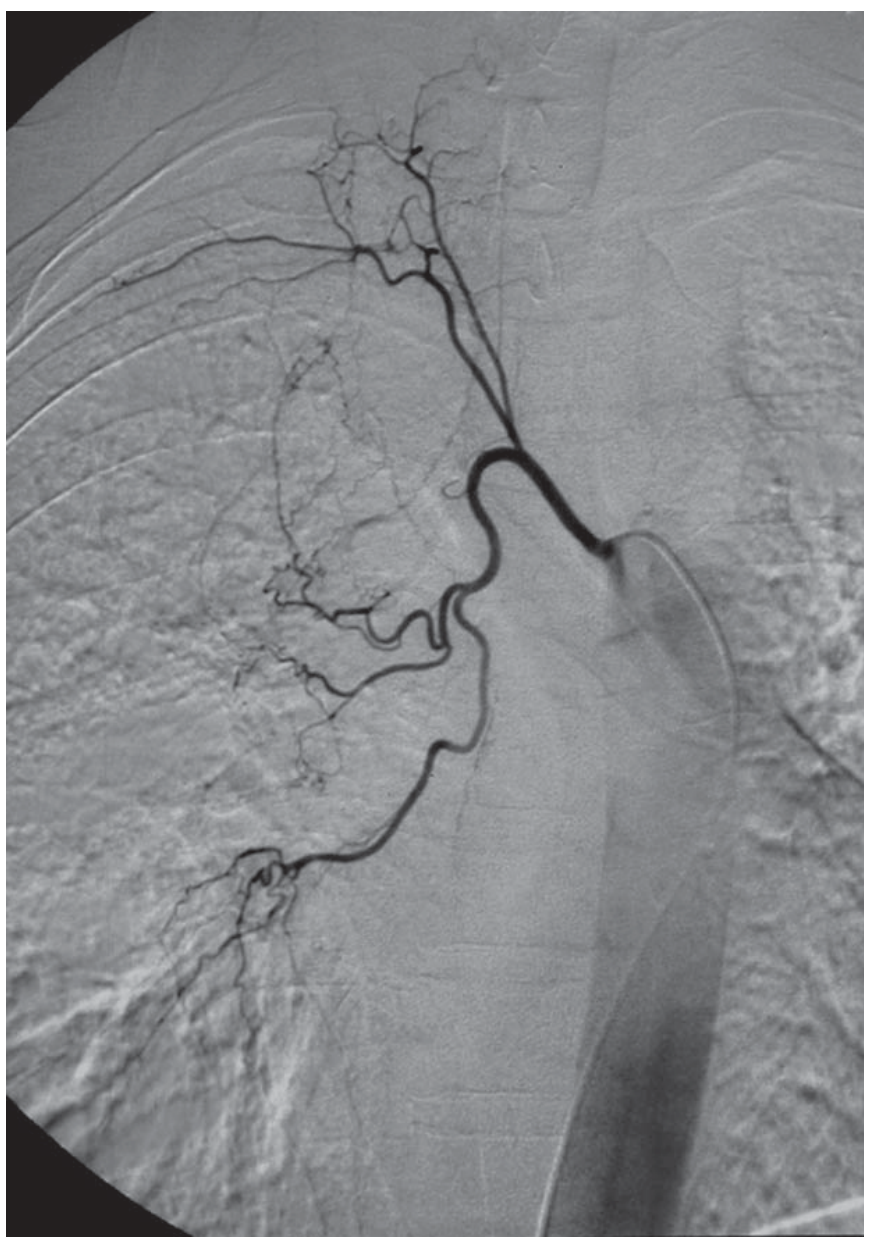

FIGURE 3. A 45-yr-old patient with a cigarette consumption of 25 pack-yrs and moderate (stage II) chronic obstructive pulmonary disease. This patient was referred for management of massive haemoptysis originating from the right upper lobe. Selective catheterisation of the right intercostobronchial trunk showing normal bronchial arteries on the side of bleeding. Immediate cessation of haemoptysis was obtained after embolisation of this artery. No late recurrence occurred during the 4-yr follow-up period.

\section{Angiographic findings}

Whereas bronchial artery catheterisation was technically successful in 34 patients, it failed in one owing to the ectopic origin of the right bronchial artery, which was found to originate from the concavity of the aortic arch on the aortogram obtained at the end of the angiographic procedure. The angiographic findings on the side of bronchial bleeding ( 23 on the right side; 12 on the left side) were available from selective arteriograms in 34 patients and from an aortogram in one patient.

On the side of bronchial bleeding, a total of 43 bronchial arteries were depicted in the 35 patients, including 16 intercostobronchial trunks, 14 common trunks from the right and left bronchial arteries and 13 isolated bronchial arteries. 29 (83\%) patients showed abnormally dilated bronchial arteries, rated as moderately dilated in 19 patients (fig. 1) and markedly dilated in 10 patients (fig. 2); six (17\%) patients had angiographically normal bronchial arteries on the side of bronchial bleeding (fig. 3). Enlarged bronchial arteries were seen with the concurrent 
TABLE 2 Comparison of angiographic findings in the 25 patients who underwent bilateral angiography

\begin{tabular}{ll}
$\begin{array}{l}\text { Angiographic aspect of bronchial arteries on the side of } \\
\text { bronchial bleeding }\end{array}$ & Angiographic aspects of bronchial arteries on the \\
\hline 25 patients & 25 patients \\
11 patients with moderately dilated bronchial arteries & 8 patients with moderately dilated bronchial arteries \\
& 3 patients with normal bronchial arteries \\
9 patients with severely dilated bronchial arteries & 6 patients with severely dilated bronchial arteries \\
& 2 patients with moderately dilated bronchial arteries \\
1 patient with normal bronchial arteries & 5 patients with normal bronchial arteries
\end{tabular}

presence of bronchial artery tortuosity in 15 patients and/or parenchymal staining with retrograde bronchial-to-pulmonary shunting in 28 patients. Angiographic features of hypervascularisation, present in $28(80 \%)$ patients were rated as moderate $(n=18)$ or marked $(n=10)$. Endobronchial bleeding during the embolisation procedure was observed in six patients.

In 25 patients, bronchial artery angiography was also obtained on the controlateral side, enabling us to compare the morphological characteristics of bronchial arteries on the ipsilateral and controlateral side of bleeding (table 2). In 19 $(76 \%)$ out of 25 patients, the angiographic findings were symmetrical; in six $(24 \%)$, abnormalities were more severe on the side of bronchial bleeding.

Table 3 summarises the angiographic findings in our cohort of patients according to the spirometric data. There was no statistically significant difference in the distribution of bronchial artery diameters (Fisher's exact test: $p=0.27$ ) and degree of hypervascularisation (Fisher's exact test: $p=0.18$ ) according to the pulmonary functional test results. No statistically significant difference was observed between the angiographic findings and the amount of haemoptysis $(p=1)$ (table 4) or between the angiographic findings and the tobacco consumption of patients $(p=0.34)$. Among the three patients in whom fibreoptic bronchoscopy had revealed endobronchial vascular abnormalities, the bronchial arteriogram was normal in one patient and showed moderate bronchial hypervascularisation in two patients.

\section{Results of the embolisation procedure}

Embolisation was technically successful in 34 patients and failed in one patient owing to the impossibility of catheterising a bronchial artery with an ectopic origin. In this latter case, bronchial bleeding stopped after medical treatment. Early results of embolisation in 34 patients were classified as follows: immediate cessation of bleeding, which was achieved in 29 (85\%) patients; and "immediate recurrence" of haemoptysis (within $48 \mathrm{~h}$ ), which was observed in five (15\%) patients. In these five patients, cessation of bleeding was obtained after a second embolisation procedure of previously occluded bronchial arteries. "Early recurrence" of bleeding $(<3$ months after endovascular treatment) was observed in five (15\%) patients; bleeding cessation was obtained by medical treatment alone $(n=2)$ or bronchial artery embolisation followed by surgery $(n=3)$ because of haemoptysis recurrence. Surgery consisted of a right upper $(n=1)$, left upper $(n=1)$ and right middle $(n=1)$ lobectomy. Two $(6 \%)$ patients presented a late recurrence of haemoptysis, 2 and 12 yrs after the initial embolisation procedure, respectively, which was successfully treated by medical treatment. From the initial study group, a total of 23 patients had no recurrence of bronchial bleeding. No statistically significant relationship was found between the recurrence of bleeding and the severity of the underlying smokingrelated bronchopulmonary disease and the angiographic aspects at the time of the embolisation procedure (table 5).

Two of the three patients with submucosal vascular abnormalities were treated by embolisation with a follow-up endoscopic examination $<48 \mathrm{~h}$ after the endovascular procedure. Fibreoptic bronchoscopy showed a complete $(n=1)$ or partial $(n=1)$ resolution of the endobronchial abnormality responsible for mild haemoptysis; in the latter case, a biopsy of the right upper lobe bronchus was obtained after the embolisation procedure.

TABLE 3 Angiographic findings according to the spirometric severity of chronic obstructive pulmonary disease (COPD)

Subjects

\begin{tabular}{|c|c|c|c|c|c|c|c|}
\hline & Subjects & \multicolumn{3}{|c|}{ Diameter of bronchial arteries } & \multicolumn{3}{|c|}{ Degree of bronchial hypervascularisation } \\
\hline Smokers with no airflow limitation & 16 & $4(25)$ & $5(37)$ & $7(44)$ & $4(25)$ & $5(31)$ & $7(44)$ \\
\hline Smokers with mild COPD & 12 & $2(16)$ & $9(74)$ & $1(8)$ & $2(16)$ & $9(74)$ & $1(8)$ \\
\hline Smokers with moderate or severe COPD & 7 & $1(14)$ & $4(58)$ & $2(28)$ & $1(14)$ & $4(58)$ & $2(28)$ \\
\hline
\end{tabular}

Data are presented as $n$ or $n(\%)$ 
TABLE 4 Amount of haemoptysis in the categories of angiographic findings

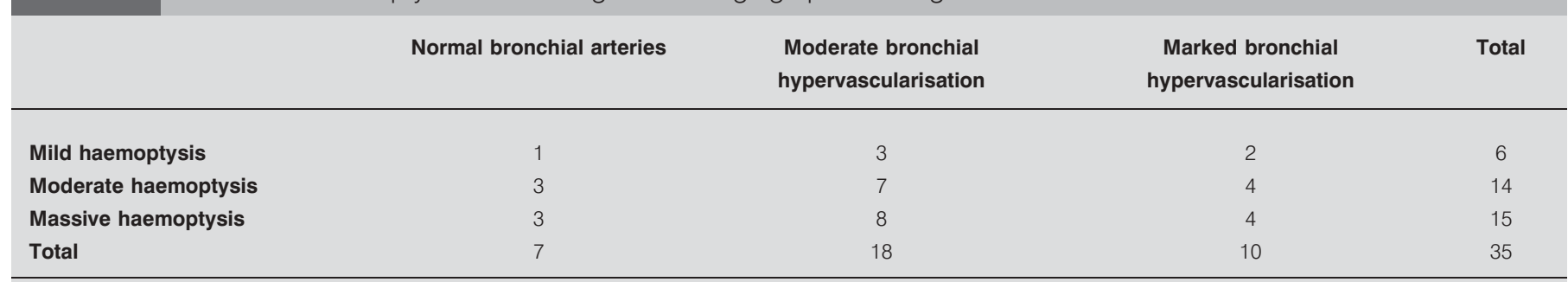

Data are presented as $n$

\section{Pathological findings}

Pathological data were available in four patients, obtained after biopsy $(n=1)$ or surgery $(n=3)$. The surgical specimens, obtained after right upper $(n=1)$, left upper $(n=1)$ and right middle $(n=1)$ lobectomy, showed dilated and numerous capillary vessels in the submucosal region of the right upper lobe bronchus. Pathological analysis revealed the presence of a submucosal angiomatous lesion at the level of the right middle lobe bronchus $(n=1)$ and dilated vessels within the mucosa of the right upper lobe bronchial walls (fig. 4), while bronchial wall vessels of the left upper lobe were described as normal in one patient. Apart from smoker's lung lesions and focal filling of alveoli by blood observed in all three patients, no associated abnormalities were described in the pathologist's reports.

\section{DISCUSSION}

To our knowledge, this is the first study describing the angiographic appearance of bronchial arteries in patients referred for smoking-related haemoptysis. This exclusive diagnosis was assessed by the absence of objective evidence of thoracic disease at the time of examination and no late diagnosis of lung cancer, the clinician's fear when haemoptysis remains of unknown origin [9]. Although strictly limited to a population referred for endovascular treatment of haemoptysis over a 22-yr period, the proportion of smoking-related haemoptysis observed in this population, i.e. $11 \%$, is comparable to those previously reported in the two studies describing cases of haemoptysis in smokers with no other comorbidity and in which there was a detailed history of the patients' smoking history [10, 11]. Over a 5-yr period, HIYAMA et al. [11] observed 51 hospitalised patients with haemoptysis, among whom six $(12 \%)$, all smokers, were considered to have cryptogenic haemoptysis according to $\mathrm{CT}$ and fibreoptic bronchoscopy findings. More recently, SAVALE et al. [10] have reported a cohort of $81(13 \%)$ patients with cryptogenic haemoptysis, diagnosed from a population of 653 consecutive patients who had been admitted to the respiratory intermediate care ward and intensive care unit for haemoptysis.

The hallmarks of our population are four-fold. First, there was a male predominance in our cohort, across which patients presented with moderate or massive hemoptysis in $83 \%$ of cases (29 out of 35 patients). This finding suggests that smokinginduced inflammatory changes can be responsible for lifethreatening haemoptysis, a situation not previously emphasised, particularly in smokers with no airflow limitation. In this latter group, usually referred to as "healthy smokers", it is noticeable that $87.5 \%$ of them (14 out of 16 patients) were referred for moderate or massive haemoptysis. Secondly, the most frequent sites of bronchial bleeding were the upper lobes ( 25 out of 35 ; $71 \%$ ) with a predominance of right-sided bronchial bleeding (18 out of $25 ; 72 \%$ ). These predominant sites of bronchial bleeding in smokers are not unexpected when they are analysed in the light of the current knowledge of the target areas of tobacco smoke. Whereas the distribution of inflammatory changes in the bronchial tree of smokers remains unknown, there is a wellknown predominant or exclusive distribution of smokinginduced inflammatory changes in the upper lung zones [14, 15].

\begin{tabular}{|c|c|c|c|c|c|c|}
\hline TABLE 5 & & Subjects & No recurrence & Immediate recurrence & Early recurrence & Late recurrence \\
\hline Subjects & & & 23 & 5 & 5 & 2 \\
\hline \multicolumn{7}{|c|}{ Pulmonary function test results } \\
\hline Smokers n & h no airflow limitation & 16 & 11 & 3 & 2 & 0 \\
\hline \multicolumn{7}{|c|}{ Diameter of bronchial arteries } \\
\hline Normal & & 7 & 6 & 0 & 1 & 0 \\
\hline Moderately & dilated & 18 & 11 & 3 & 3 & 1 \\
\hline Severely $d$ & ated & 10 & 6 & 2 & 1 & 1 \\
\hline
\end{tabular}

Data are presented as n. 

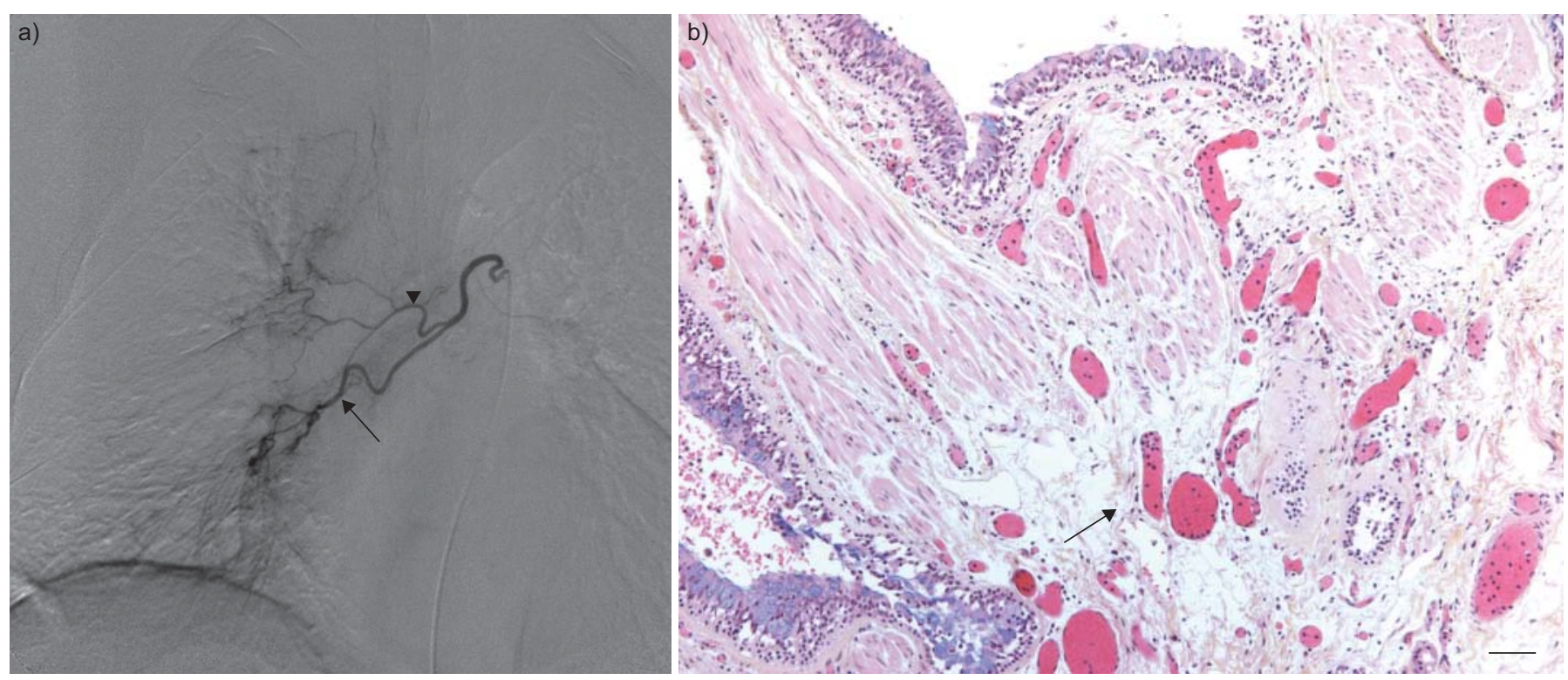

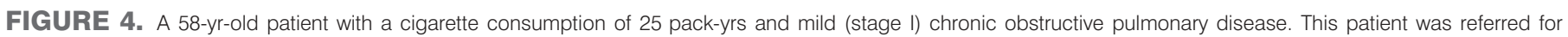

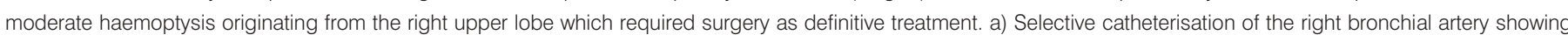

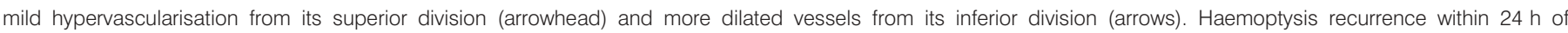

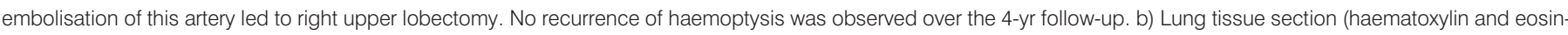
safran) demonstrating numerous and dilated capillary vessels (arrow) in a cartilaginous bronchial wall of the right upper lobe. Scale bar $=200 \mu \mathrm{m}$

Thirdly, moderate to marked bronchial hypervascularisation was found in $80 \%$ (28 out of 35 patients) of our study group. When bilateral angiograms were available, the angiographic appearance of bronchial arteries was symmetrical in $76 \%$ of cases (19 out of 25 patients). These results suggest the presence of underlying bronchial hyperaemia, which was confirmed by the pathological findings available for four of our patients. Hypervascularisation within the bronchial wall remains an ill-defined process in the pathogenesis of asthma and chronic airway inflammation, such as COPD [16, 17]. As previously underlined by several authors, there is a remarkable proliferative capacity of the bronchial vessels in response to a variety of pulmonary diseases $[18,19]$. In a recent study, НАSHIMOTO et al. [20] reported that submucosal vascularity in patients with COPD was greater than in control subjects, but not significantly, except for the proportion of vascularity in the small airways. These data are consistent with the results of previous studies [21]. The vessel increase in COPD patients might have occurred as the result of simple tobaccoinduced inflammation but angiogenic factors might also be secreted as an asthma-specific inflammation. In a minority of patients, more pronounced vascular lesions were observed at the site of bleeding, as suggested by the angiographic findings of more severe hypervascularisation in six patients with bilateral angiograms. In the context of angiogenesis in chronic bronchitis, one may also reconsider the endobronchial vascular lesions depicted in $9 \%$ of our study group (three out of 35 patients). Whereas they resemble the endobronchial findings described in Dieulafoy's disease, one may also raise the hypothesis that such lesions could be acquired. Among the 12 cases of Dieulafoy's disease reported in the literature, five were described in smokers who had no associated comorbidity [22-30].

The fourth hallmark of our study group is the lack of relationship between the angiographic findings and the risk of bronchial bleeding and the severity of the underlying bronchopulmonary disease. Whereas most patients had various degrees of bronchial hypervascularisation, $20 \%$ of our population (seven out of 35 patients) had angiographically normal bronchial arteries on the side of bronchial bleeding. The immediate cessation of bleeding after bronchial embolisation confirms the responsibility of angiographically normal bronchial arteries in such cases. With regard to the efficacy of embolisation in the overall population, an immediate cessation of bleeding was obtained in $85 \%$ of cases ( 25 out of 34 patients), which is in the range of the immediate control of bleeding reported in the literature in a variety of underlying diseases [31-34]. An early recurrence of bleeding was observed in five $(15 \%)$ out of 34 patients, while only two patients had a late recurrence of bleeding (two out of $34 ; 6 \%$ ). As in many varieties of chronic disorders, this is likely to reflect the recruitment of blood supply and revascularisation by the underlying bronchial inflammation or progression of the underlying disease [34]. Classifying our cohort of smokers according to the revised spirometric classification recently proposed in the GOLD strategy, we failed to demonstrate any statistically significant relationship between the angiographic findings and the COPD severity, which is in agreement with previous reports. In a microradiographic study of 17 patients with emphysema and chronic bronchitis, TURNER-WARWICK [35] has observed that bronchial artery enlargement was not clearly related to the presence of associated bronchitis. In a more recent study, KUWANO et al. [21] found no correlation between the degree of vascularity and airflow limitation in patients with COPD. Moreover, we did not find any relationship between the risk of bronchial bleeding recurrence and the angiographic aspect of bronchial arteries at the time of the embolisation procedure. 
Several limitations should be acknowledged in this study. The criteria for excluding any specific anatomic lesion potentially responsible for haemoptysis did not include chest $\mathrm{CT}$ in nine patients who were referred prior to the advent of CT technology. However, these patients had been investigated by conventional bronchography and/or pulmonary tomography which did not reveal any abnormality. In addition, the uneventful mean follow-up of 9.72 yrs reported for these nine patients reinforces the diagnosis of cryptogenic haemoptysis considered at the time of initial management. The second limitation concerns the exclusion of lung cancer in the 10 patients with a follow-up of $<2$ yrs. It is important to emphasise that these patients correspond to the most recently referred to our institution; subsequently, they benefited from fibreoptic bronchoscopy and chest CT, not only at the time of diagnosis, but also in their follow-up. The absence of endobronchial abnormality and normal chest CT scans in their follow-up reinforce the likelihood of cryptogenic haemoptysis. Lastly, two additional limitations should be outlined, dealing with the lack of control group and the length of the inclusion period. Concerning the control group, we did not encounter more than a few cases of nonsmokers presenting with cryptogenic haemoptysis over the inclusion period, which precluded any structured comparison with the studied population. The length of the inclusion period, $22 \mathrm{yrs}$, was related to the relative rarity of the target population that shared three similar criteria, namely the symptoms, the underlying cause and the therapeutic option.

In conclusion, our study demonstrates that tobacco smoke and its bronchopulmonary inflammatory consequences represent an aetiology for bronchial bleeding, independent of the severity of the underlying disease.

\section{STATEMENT OF INTEREST}

None declared.

\section{REFERENCES}

1 Johnston H, Reisz G. Changing spectrum of hemoptysis. Underlying causes in 148 patients undergoing diagnostic flexible fiberoptic bronchoscopy. Arch Intern Med 1989; 149: 1666-1668.

2 Santiago S, Tobias J, Williams AJ. A reappraisal of the causes of hemoptysis. Arch Intern Med 1991; 151: 2449-2451.

3 McGuinness G, Beacher J R, Harkin TJ, et al. Hemoptysis: prospective high resolution $\mathrm{CT} /$ bronchoscopic correlation. Chest 1994; 105: 1155-1162.

4 Alaoui AY, Barial M, Boutahiri A, et al. Clinical characteristics and etiology of hemoptysis in a pneumology service: 291 cases. Rev Mal Respir 1992; 9: 295-300.

5 Hirshberg B, Biran I, Glazer M, et al. Hemoptysis: etiology, evaluation and outcome in a tertiary referral hospital. Chest 1997; 112: $440-444$.

6 Abal AT, Nair PC, Cherian J. Haemoptysis: aetiology, evaluation and outcome - a prospective study in a third-world country. Respir Med 2001; 95: 548-552.

7 Adelman M, Haponik EF. Cryptogenic hemoptysis. Clinical features, bronchoscopic findings and natural history in 67 patients. Ann Intern Med 1985; 102: 829-834.

8 Boulay F, Berthier F, Sisteron O, et al. Seasonal variation in cryptogenic and noncryptogenic hemoptysis hospitalizations in France. Chest 2000; 118: 440-444.
9 Herth F, Ernst A, Becker HD. Long-term outcome and lung cancer incidence in patients with hemoptysis of unknown origin. Chest 2001; 120: 1592-1594.

10 Savale L, Parrot A, Khalil A, et al. Cryptogenic hemoptysis. From a benign to a life-threatening pathologic vascular condition. Am J Respir Crit Care Med 2007; 175: 1181-1185.

11 Hiyama J, Horita N, Shiota Y, et al. Cryptogenic hemoptysis and smoking. Chest 2002; 121: 1375-1376.

12 Remy J, Remy-Jardin M, Voisin C. Endovascular management of bronchial bleeding. In: Buttler J, ed. The Bronchial Circulation. New York, Marcel Dekker, 1992; pp. 667-723.

13 Global strategy for the diagnosis, management, and prevention of chronic obstructive pulmonary disease. GOLD Executive Summary. Am J Respir Crit Care Med 2007; 176: 532-555.

14 Soejima K, Yamaguchi K, Kohda E, et al. Longitudinal follow-up of smoking-induced lung density changes by high-resolution computed tomography. Am J Respir Crit Care Med 2000; 161: 1264-1273.

15 Remy-Jardin M, Edme JL, Boulenguez C, et al. Longitudinal follow-up study of smoker's lung with thin-section CT in correlation with pulmonary function tests. Radiology 2002; 222: 261-270.

16 McDonald D. Angiogenesis and remodelling of airway vasculature in chronic inflammation. Am J Resp Crit Care Med 2001; 164: 539-545.

17 Jeffery PK. Remodeling in asthma and chronic obstructive lung disease. Am J Respir Crit Care Med 2001; 164: S28-S38.

18 Charan NB, Baile EM, Pare PD. Bronchial vascular congestion and angiogenesis. Eur Respir J 1997; 10: 1173-1180.

19 Widdicombe JG. Possible vascular mechanisms in COPD. Eur Respir Rev 1997; 7: 151-153.

20 Hashimoto M, Tanaka H, Abe S. Quantitative analysis of bronchial wall vascularity in the medium and small airways of patients with asthma and COPD. Chest 2005; 127: 965-972.

21 Kuwano K, Bosken CH, Pare PD, et al. Small airway dimensions in asthma and in chronic obstructive pulmonary disease. Am Rev Respir Dis 1993; 148: 1220-1225.

22 Sweerts M, Nicholson AG, Goldstraw P, et al. Dieulafoy's disease of the bronchus. Thorax 1995; 50: 697-698.

23 Van der Werf, Timmer A, Zjlstra JG, Fatal haemorrhage from Dieulafoy's disease of the bronchus. Thorax 1999; 54: 184-185.

24 Stoopen E, Baquera-Heredia J, Cortes D, et al. Dieulafoy's disease of the bronchus in association with a paravertebral neurilemoma. Chest 2001; 119: 292-294.

25 Hope-Gill B, Prathibha BV. Bronchoscopic and angiographic findings in Dieulafoy's disease of the bronchus. Hosp Med 2002; 63: 178-179.

26 Bathia P, Hendy MS, Li-Kam-Wa E, et al. Recurrent embolotherapy in Dieulafoy's disease of the bronchus. Can Respir J 2003; 10: 331-333.

27 Kuzucu A, Gürses I, Soysal O, et al. Dieulafoy's disease: a cause of massive hemoptysis that is probably underdiagnosed. Ann Thorac Surg 2005; 80: 1126-1128.

28 Loschhorn C, Nierhoff N, Mayer R, et al. Dieulafoy's disease of the lung: a potential disaster for the bronchoscopist. Respiration 2006; 73: 562-565.

29 Sheffield EA, Moore-Gillon J, Murday AR, et al. Massive hemoptysis caused by spontaneous rupture of a bronchial artery. Thorax 1988; 43: 71-72.

30 Soda H, Oka M, Kohno S, et al. Arteriovenous malformation of the bronchial artery showing endobronchial protrusion. Intern Med 1995; 34: 797-800.

31 Cremaschi P, Nascimbene C, Vitulo P, et al. Therapeutic embolization of bronchial artery: a successful treatment in 209 cases of relapse hemoptysis. Angiology 1993; 44: 295-299.

32 Rabkin JE, Astafjev VI, Gothman LN, et al. Transcatheter embolization in the management of pulmonary hemorrhage. Radiology 1987; 163: 361-365. 
33 Kato A, Matsumoto K, Fukahori T, et al. Bronchial artery embolization for hemoptysis due to benign diseases: immediate and long-term results. Cardiovasc Intervent Radiol 2000; 23: 351-357.
34 Swanson KL, Johnson CM, Prakash UBS, et al. Bronchial artery embolization. Experience with 54 patients. Chest 2002; 121: 789-795.

35 Turner-Warwick M. Systemic arterial patterns in the lung and clubbing of the fingers. Thorax 1963; 18: 238-250. 